\title{
Identification and Characterization of the Most Abundant Cellulases in Stylet Secretions from Globodera rostochiensis
}

\author{
Sajid Rehman, Patrick Butterbach, Herman Popeijus, Hein Overmars, Eric L. Davis, \\ John T. Jones, Aska Goverse, Jaap Bakker, and Geert Smant
}

First, second, third, fourth, seventh, eighth, and ninth authors: Laboratory of Nematology, Wageningen University, Binnenhaven 5, 6709PD Wageningen, the Netherlands; fifth author: Department of Plant Pathology, North Carolina State University, Campus Box 7903, Raleigh, NC 27695-7616; and sixth author: Plant Pathogen Interactions Programme, Scottish Crop Research Institute, Dundee, DD2 5DA, United Kingdom.

Accepted for publication 12 September 2008.

\begin{abstract}
Rehman, S., Butterbach, P., Popeijus, H., Overmars, H., Davis, E. L., Jones, J. T., Goverse, A., Bakker, J., and Smant, G. 2009. Identification and characterization of the most abundant cellulases in stylet secretions from Globodera rostochiensis. Phytopathology 99:194-202.

Plant-parasitic cyst nematodes secrete cell wall modifying proteins during their invasion of host plants. In this study, we used a monoclonal antibody to immunopurify and to sequence the $\mathrm{N}$ terminus of the most abundant cellulases in stylet secretions of preparasitic juveniles of Globodera rostochiensis. The N-terminal amino acid sequence perfectly matched the sequence of an expressed sequence tag of two nearly identical genes, named Gr-eng3 and Gr-eng4, which show relatively low simi-

cellulose, indicating that these genes encode active cellulases. To date, the cellulases in cyst nematodes are comprised of three possible domain structure variants with different types of ancillary domains at the $\mathrm{C}$ terminus of the glycosyl hydrolase family 5 (GHF5) domain. We used Bayesian inference to show that the phylogeny of the GHF5 domain based on currently available data suggest that the extant nematode cellulases arose through reshuffling of the GHF5 domain with different types of ancillary domains as relatively independent units. Knocking-down $\mathrm{Gr}$ eng3 and Gr-eng4 using RNA interference resulted in a reduction of nematode infectivity by $57 \%$. Our observations show that the reduced infectivity of the nematodes can be attributed to poor penetration of the host's root system at the onset of parasitism.
\end{abstract} larity with the previously identified Gr-eng1 and Gr-eng2 (i.e., 62\% similarity and $42 \%$ identity). The recombinantly produced proteins from Gr-eng3 and Gr-eng4 demonstrated specific activity on carboxymethyl-
Additional keywords: $\beta-1,4-$ endoglucanase, cell wall degradation, plantnematode interactions.
Cellulose is made of linear chains of $\beta$-1-4-linked D-glucose and constitutes the most abundant natural carbohydrate polymer on earth. In plants, stacked sheets of these parallel $\beta$-glucan polymers interconnect along their length with hydrogen bonds to form insoluble microfibrils. The microfibrils assemble into the cellulose network, which represents the main scaffolding structure in plant cell walls. The cellulose scaffold is reinforced by hemicelluloses, such as xyloglucans and glucuronarabinoxylans. This (hemi)cellulose composite is embedded in a matrix of pectic polysaccharides and lignins, which collectively provide plant cells with a strong constitutive protective armor against invading parasites $(4,5)$.

Sedentary plant-parasitic nematodes penetrate roots of host plants close to the root apex and migrate through the root tissues until they settle and induce the formation of an elaborate feeding site on which they depend for all nutrients required for development to the adult stage. Cyst nematodes migrate intracellularly, breaking down cell walls as they progress from cell to cell, whereas root knot nematodes migrate intercellularly, softening

Corresponding author: G. Smant; E-mail address: geert.smant@wur.nl

GenBank accessions: Gr-eng3, AF408155 (mRNA) and AF408154 (gene); Greng4, AF408156 (mRNA) and AF408157 (gene).

* The $e$-Xtra logo stands for "electronic extra" and indicates that there is an additional figure online showing the genomic organization of $\mathrm{Gr}$-eng3 and $\mathrm{Gr}$ eng4. Figures 5 and 6 appear in color online.

doi:10.1094/PHYTO-98-2-0194

(C) 2009 The American Phytopathological Society cell walls to aid their migration. During migration of cyst nematodes, the cell walls are broken down by strong outward thrusts of the oral stylet of the nematode. Migration is further facilitated by the release of cell wall degrading enzymes (e.g., cellulases, pectate lyases, polygalacturonases, and endoxylanases) from the stylet lumen, which is connected to three esophageal gland cells $(10,13,19,20,24,27,29,31,32)$. Recent studies have shown that in addition to the conventional enzymes listed above, cyst nematodes also secrete $\beta$-expansins $(16,21)$, which are believed to disrupt noncovalent interactions between cell wall polymers, making them more susceptible to the activity of conventional enzymes.

The first nematode cell wall degrading enzymes-cellulaseswere identified in two cyst nematode species (27). A specific antibody (MGR48) directed toward at least three proteins in the stylet secretions of the potato cyst nematode Globodera rostochiensis was used to purify two corresponding antigens from nematode homogenates. Following $\mathrm{N}$-terminal amino acid sequencing, two cellulase genes, named Gr-eng1 and Gr-eng2 and coding for proteins of 49 and $39 \mathrm{kDa}$, respectively, were cloned using degenerate oligonucleotide primers and polymerase chain reaction (PCR)-based cloning (27). The third protein recognized by MGR48 was refractory to amino acid sequencing, and consequently, its corresponding gene could not be characterized. However, in earlier experiments this smaller protein proved to be the most abundant antigen in stylet secretions of the preparasitic juveniles (26). Here, we describe the characterization of two genes (Gr-eng3 and Gr-eng4) encoding the 32-kDa antigen recognized by MGR48 using a modified protocol for N-terminal protein sequencing and $5^{\prime}$-end expressed sequence tags. The genes 
code for novel cellulases comprising a glycosyl hydrolase family 5 (GHF5) type of catalytic domain.

The group of GHF5 cellulases in plant-parasitic nematodes consists of several protein domain structure variants. The largest variant (e.g., Gr-engl) includes the GHF5 catalytic domain, a linker, and a cellulose binding domain, while the novel genes described in this paper only include a GHF5 domain. The third and intermediate variant (e.g., Gr-eng2) includes a GHF5 domain and a short ancillary C-terminal domain. It has been suggested that these extant domain structure variants may have evolved by a sequential loss of cellulose binding domain and linker sequences (17). In this paper, we test this sequential loss model using phylogeny inference of the domain structure variants in currently available cyst nematode cellulases.

\section{MATERIALS AND METHODS}

Nematodes. Dried cysts of $G$. rostochiensis pathotype Ro1 Mierenbos were soaked on a $100 \mu \mathrm{m}$ sieve in potato root diffusate to collect hatched preparasitic second-stage juveniles (J2s) (7). Freshly hatched $\mathrm{J} 2 \mathrm{~s}$ in suspension were mixed with an equal volume of $70 \%$ (wt/vol) sucrose in a centrifuge tube and covered with a layer of sterile tap water. Following centrifugation for $5 \mathrm{~min}$ at $1,000 \times g$, juveniles were collected from the sucrosewater interface with a Pasteur pipette and washed three times with sterile tap water. Subsequently, the J2s were either used for experiments directly or stored at $-80^{\circ} \mathrm{C}$ until further use.

DNA cloning and analysis. An oligo-dT-primed cDNA library from $G$. rostochiensis J2s was prepared in the vector pcDNAII (Invitrogen, San Diego). Individual colonies plated on LuriaBertani medium supplemented with ampicillin were randomly picked and grown in 1-ml liquid cultures. Plasmid DNA was isolated from the cells following overnight growth at $37^{\circ} \mathrm{C}$. The 5 '-end expressed sequencing tags were subsequently generated using cycle sequencing with dye terminator chemistry $(20,27)$. The single read sequences were analyzed using the program Basic Local Alignment Search Tool algorithm (TBLASTN, BLASTN, and BLASTP) with default settings at the National Center for Biotechnology Information (available online). One expressed sequence tag (named GE1985) encoded a partial open reading frame that showed similarity to cellulases of various origins. The sequence similarity with other nematode cellulases, however, was only slightly higher than those found in other organisms. The sequence downstream of EST GE1985 was identified using the M13 universal sequencing primer (Table 1). A start codon was missing in GE1985; therefore, a primer (Eng-r1; Table 1) was designed to amplify the region upstream of the tag when combined with a plasmid derived primer (PCDNA5-f1; Table 1) in a PCR using a plasmid prep of the cDNA library. To confirm the integrity of the cDNA that could be deduced from the amplified fragments, two primers (Eng-f1 and Eng-r2; Table 1) were combined in a similar PCR that produced a single band of 1,194 bp. This band was cloned into the pCR2.1-TOPO (Invitrogen) and subjected to DNA sequencing. A similar PCR with the primers Eng-f1 and Eng-r2 was performed using genomic DNA as template, which was extracted from hatched $\mathrm{J} 2 \mathrm{~s}$ by alkaline/sodium dodecyl sulfate (SDS) lysis and phenol/chloroform extraction (28). The amplified genomic fragment was cloned into pCR2.1TOPO vector and sequenced as described above.

The program Signal-P was used to predict the presence of a signal peptide consensus sequence (available online). Protein patterns and domains in the predicted open reading frames were identified using the PFAM analysis tool as provided on the worldwide web.

In situ hybridization. DNA probes were amplified from clone GE1985 using the oligonucleotide primers Eng-insitu-f and Enginsitu-r (Table 1) and digoxigen-11-dUTP. J2s were fixed overnight in $2 \%$ paraformaldehyde, cut into sections, and permeabilized as described $(8,27)$. Fixed sections were then incubated at $50^{\circ} \mathrm{C}$ with sense or antisense DNA probes followed by digestion with RNase A and stringent washes. Hybridized DNA probe was detected using an antidigoxigenin antibody and alkaline phosphatase staining (Genius kid, Boehringer Mannheim). Stained J2s were examined with differential interference contrast microscopy (Leica, Deerfield).

Heterologous expression and activity assay. The open reading frames of Gr-eng-3 and Gr-eng-4 without the predicted Nterminal signal peptides (nucleotides 77 to 1015) were subcloned into the pET101/D-TOPO expression vector (Invitrogen) using the oligonucleotide primers Eng-pETf and Eng-pETr (Table 1). The plasmids were introduced into Escherichia coli strain TOP10 (Invitrogen) to sequence the constructs. Recombinant plasmid DNA was introduced into E. coli strain BL21Star (DE3) for expression (Invitrogen). Expression of the recombinant protein was determined on SDS-polyacrylamide gel electrophoresis (PAGE) with Coomassie brilliant blue staining and western blotting following induction of the transformants with $1 \mathrm{mM}$ isopropyl $\beta$ D-thiogalactoside (25).

Carboxymethylcellulose (CMC) was used as a substrate in a cup plate assay to determine CMC hydrolyzing activity of

TABLE 1. Oligonucleotide primers used for amplification of nematode cDNA (fragments) and genomic DNA ${ }^{\mathrm{a}}$

\begin{tabular}{ll}
\hline Name & Primer sequence $\left(5^{\prime} \rightarrow 3^{\prime}\right)$ \\
\hline Eng-f & GAATGTGCGCTTTGATTTATG \\
Eng-r1 & GCACAACATTGCTGTTACAGC \\
Eng-r2 & CTAAGCTTTGATTTATTCACCTTTC \\
Eng-pETf & CACCGTCACAGCCCTCCCTAT \\
Eng-pETr & ACCGCGGCAACTTACT \\
Eng-insitu-f & CCGCGGAATATGCCAAAATGAAG \\
Eng-insitu-r & CCGGCGTAAAAGGCAATGTGTATG \\
M13 universal primer $(-20)$ & GTAAAACGACGGCCAGT \\
PCDNA5-f & GGTGACACTATAGAATACTCAAGCTATGCA \\
T7ENG3-f & TAATACGACTCACTATAGGCGATGAAATCAATTCTGCTAAA \\
T7ENG3-r & TAATACGACTCACTATAGGGATTGTTTGCCTCATTTTTG \\
T7A41-f & TAATACGACTCACTATAGGGAAACTCGCGTGGGATTCC \\
T7A41-r & TAATACGACTCACTATAGGGCCCATTGTGTGTGTAAATG \\
RT-eng1f & CTGCACATTGTGTTCTGTAACGC \\
RT-eng1r & TTGAGACAACAGTGACGCT \\
RT-eng2r & CAGCGGCAGGTTTGGCCGGAGG \\
RT-eng3f & GATCATTCTAAGATCGGCG \\
RT-eng3r & GGTTGCAAATGTTGTTGATT \\
RT-A41f & CAAACTGATGCTTCGCCAA \\
RT-A41r & TGAATTTGAATGGTTTCGTGC \\
\hline
\end{tabular}

${ }^{\mathrm{a}}$ Underlined is the $\mathrm{T} 7$-promotor sequence to prepare template for in vitro transcription. 
cellulase. CMC $(0.2 \%$, wt/vol) was dissolved in a phosphatecitrate buffer $\left(50 \mathrm{mM} \mathrm{K}_{2} \mathrm{HPO}_{4}\right.$ adjusted to $\mathrm{pH} 7.4$ with $1 \mathrm{M}$ citric acid) with $0.5 \%(\mathrm{wt} / \mathrm{vol})$ agarose. CMC plates were incubated overnight at $30^{\circ} \mathrm{C}$ and stained with $0.02 \%$ Congo red (18).

$\mathbf{N}$-terminal protein sequencing. Four protein bands $(31,32$, 39 , and $49 \mathrm{kDa}$ ) were detected with the monoclonal antibody MGR48 on western blots of homogenates from G. rostochiensis. The protein band of $32 \mathrm{kDa}$ was purified from homogenates of $G$. rostochiensis $\mathrm{J} 2 \mathrm{~s}$ using continuous flow SDS-PAGE followed by fraction identification and immunoaffinity chromatography with monoclonal antibody MGR48 (27). The purified fraction was rerun on an analytical SDS-PAGE gel utilizing a Tris-tricine buffer system and blotted onto a polyvinylidene fluoride membrane for N-terminal sequencing (ARIAD Pharmaceuticals, Cambridge, MA). The sequence analysis of the protein band showed multiple $\mathrm{N}$ termini including one major sequence. Additional runs using the reagent o-phtalaldehyde at the fourth cycle blocked all $\mathrm{N}$ termini except the major sequence and resulted in a peptide sequence of 26 amino acids. The 23rd and the 26th cycle in the Edman's degradation procedure did not yield an unambiguous reading of one of the residues in the high-performance liquid chromatography.

RNA interference by soaking. For knocking-down expression of Gr-eng3 and Gr-eng4 by soaking in gene-specific doublestranded (ds)RNA, two primers were used, T7ENG3-f and T7ENG3-r (Table 1), to generate a dsRNA fragment that targeted the region from nucleotides 403 to 803 . This region is identical between $G r$-eng3 and Gr-eng4. Similarly, the primers T7A41-f and T7A41-r were used to generate control dsRNA. The T7 promoter sequence was included in the sequence of the primers (underlined). RNA was transcribed in vitro using T7 RNA polymerase to prepare dsRNA at $2 \mathrm{mg} / \mathrm{ml}$ for soaking of the nematodes (Ambion, Austin). A typical soaking reaction included 10,000 nematodes in a dsRNA solution $(2 \mathrm{mg} / \mathrm{ml}$ dsRNA, $3 \mathrm{mM}$ Spermidine, and $0.05 \%$ gelatin) for $40 \mathrm{~h}$. Control nematodes were soaked in solutions without dsRNA or with dsRNA from genes not related in $G$. rostochiensis. From each reaction, 8,000 J2s were used for reverse transcription (RT)-PCR, while 2,000 J2s were used for the nematode infection assay. The viability of the nematodes was checked by examination under a microscope after soaking in dsRNA and after sterilization for the nematode infection assay. The soaking experiment with dsRNA was repeated three times.

For semiquantitative RT-PCR, total RNA was extracted from nematodes using TRIzol reagent and transcribed into cDNA using Superscript II reverse transcriptase (Invitrogen). RT-PCR was performed as described by Chen et al. (6) using the primer combinations RT-eng3f and RT-eng3r for Gr-eng3, RT-eng1f and RT-eng1r for Gr-eng1, RT-eng1f and RT-eng2r for Gr-eng2, and RT-A41f and RT-A41r for Gr-A41 (Table 1) designed outside the region targeted by the dsRNA. Aliquots of reaction were removed after 26 cycles and visualized on $1 \%$ agarose gel stained with ethidium bromide (25).

N-terminus
N-term. Gr-ENG3/4
N-term. Gr-ENG1
N-term. Gr-ENG2
Identical/similar

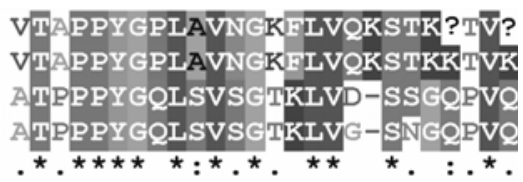

Fig. 1. An alignment of the N-terminal amino acid sequences of the $32-\mathrm{kDa}$ protein immunopurified from nematode homogenates using MGR48 (N terminus), from the predicted open reading frame in Gr-eng3/4 (N-term. GrENG3/4), and from Gr-ENG1 (N-term. Gr-ENG1) and Gr-ENG2 (N-term. GrENG2). The alignment was generated in ClustalW1.8 using the GONNET matrix to discover identical (*), conserved (:), and semiconserved (.) amino acids. - marks a gap introduced in the sequences in ClustalW, while a ? in the peptide sequence indicates an unresolved residue in the high-performance liquid chromatography analysis of the products following Edman's biochemistry.
For the nematode infection assay, internodal cuttings were taken from Solanum tuberosum LineV, grown in sterile culture in $12 \mathrm{~cm}^{2}$ plates containing MS20 media. For each treatment, four plates with four internodal cuttings on each plate were inoculated with 175 nematodes. The nematodes were surface-sterilized prior to inoculation (11). Nematode penetration of the roots was assessed with a binocular microscope at $24 \mathrm{~h}$ postinoculation. Roots were taken from the plates for acid fuchsin staining (3). The percentage of nematodes inside the roots was calculated as a proportion of the total number of nematodes inoculated. A distinction was made between partial penetration of the root for nematodes that had part of their body still outside the root epidermis. Full penetration was scored only when a nematode completely passed the epidermis. The statistical analysis of the data was done using the Student's $t$ test for two samples assuming equal variances.

Phylogenetic analysis. The nonredundant protein database at NCBI was queried with the Gr-ENG3/4 sequence using the BLASTP algorithm. All matching cellulase sequences from cyst nematode species were subsequently trimmed in order to include the GHF5 domain in the analysis (i.e., Gr-ENG1, GenBank identification number (gi): 2624931; Gr-ENG2, gi: 2654525; HgENG1, gi: 2257849; Hg-ENG2, gi: 2257951; Hg-ENG3, gi: 3513544; Hg-ENG4,gi: 15822650; Hg-ENG5, gi: 33151119; HgENG6, gi: 30348356; Hs-ENG1, gi: 10800865; Hs-ENG2, gi: 10800867; Gts-ENG1, gi: 5923869; Gts-ENG2, gi:5923871). One matching cellulase (Mi-ENG1, gi: 563966) from Meloidogyne incognita was used as outgroup. A multiple sequence alignment with 15 protein sequences was made in ClustalW version 1.83 at the European Bioinformatics Institute online server using the GONNET250 substitution matrix. The alignment was manually optimized and gaps were removed. In total, 328 informative positions were used for phylogenetic inference using Bayesian analysis (MrBayes version 3.1.2) (22). The analysis was run with a mixed model of amino acid evolution to allow model-jumping for fixed-rate models for four runs with four chains each for $1,000,000$ generations. The first 25,000 generations of the run were discarded as burn-in. In the resulting rooted phylogeny, the nodes with a posterior probability lower than 0.95 are considered to be unresolved.

\section{RESULTS}

Preparative SDS-PAGE was used to isolate a protein fraction ranging from 30 to $35 \mathrm{kDa}$ from homogenates of 5 million preparasitic $\mathrm{J} 2 \mathrm{~s}$ of $G$. rostochiensis. A $32-\mathrm{kDa}$ protein band was purified from this fraction using monoclonal antibody MGR48. The purified protein was rerun on an analytical SDS-PAGE for western blotting. Five micrograms of blotted protein was subsequently used for $\mathrm{N}$-terminal amino acid sequencing, which resulted in one major sequence of 26 amino acids with two uncertainties because of the lack of an unambiguous signal at positions 23 and 26 (question marks in Fig. 1).

Database searches with the TBLASTN algorithm using the Nterminal sequence of the purified 32-kDa antigen of MGR48 yielded one nearly perfect matching nucleotide stretch in one expressed sequence tag (library clone GE1985) from G. rostochiensis. All residues that had been resolved reliably in the $\mathrm{N}$ terminus of the antigen of MGR48 were identical to the protein sequence predicted by GE1985. The two residues that were still uncertain in the $\mathrm{N}$-terminal sequence also did not match the predicted sequence in the expressed sequence tag. Further sequencing of the clone from which the tag originated revealed that a cDNA insert of 1,196 bp was present, including a region encoding an open reading frame of 337 amino acids. The first 24 amino acids at the $\mathrm{N}$ terminus of the open reading frame were predicted to be part of a signal peptide for secretion using the Signal-P 3.0 software. The sequence of the $\mathrm{N}$ terminus of the purified $32-\mathrm{kDa}$ protein starts exactly where Signal-P software predicts the 
cleavage site of the signal peptide for secretion in the protein encoded by clone GE1985.

While sequencing constructs for the recombinant expression of the open reading frame in clone GE1985, we found two highly similar cDNA molecules differing in sequence at eight nucleotide positions. The predicted proteins of these cDNA molecules both have identical matches with the $\mathrm{N}$ terminus of the purified MGR48 antigen. The expression clones were constructed by PCR with forward and reverse primers designed to amplify the coding sequence without signal peptide for secretion and stop codon, respectively. Consequently, the polymorphisms detected in the two cDNAs could therefore reflect errors randomly introduced by DNA polymerases. In order to test this, we amplified and sequenced the corresponding coding sequences from genomic DNA of $G$. rostochiensis. All eight nucleotide differences were also found in the corresponding genomic fragments, making it very unlikely that they are all artifacts caused by PCR errors. Therefore, the epitope of MGR48 is present in two nearly identical proteins, hereafter named Gr-ENG3 and Gr-ENG4. The theoretical molecular masses of Gr-ENG3 and Gr-ENG4 are 35,388 and $35,378 \mathrm{Da}$, respectively, with predicted isoelectric points of 8.67 and 8.80. The genomic regions coding for the GHF5 domain in Gr-eng3 and Gr-eng4 are interspersed with six introns. It is noted that Gr-eng3 and Gr-eng4 have one uniquely used intron position (position II) and five intron positions (positions I, III, IV, VI, and VIII) in common with Gr-eng1 and Gr-eng2, with which they share $42 \%$ identity and $62 \%$ similarity across the coding region.
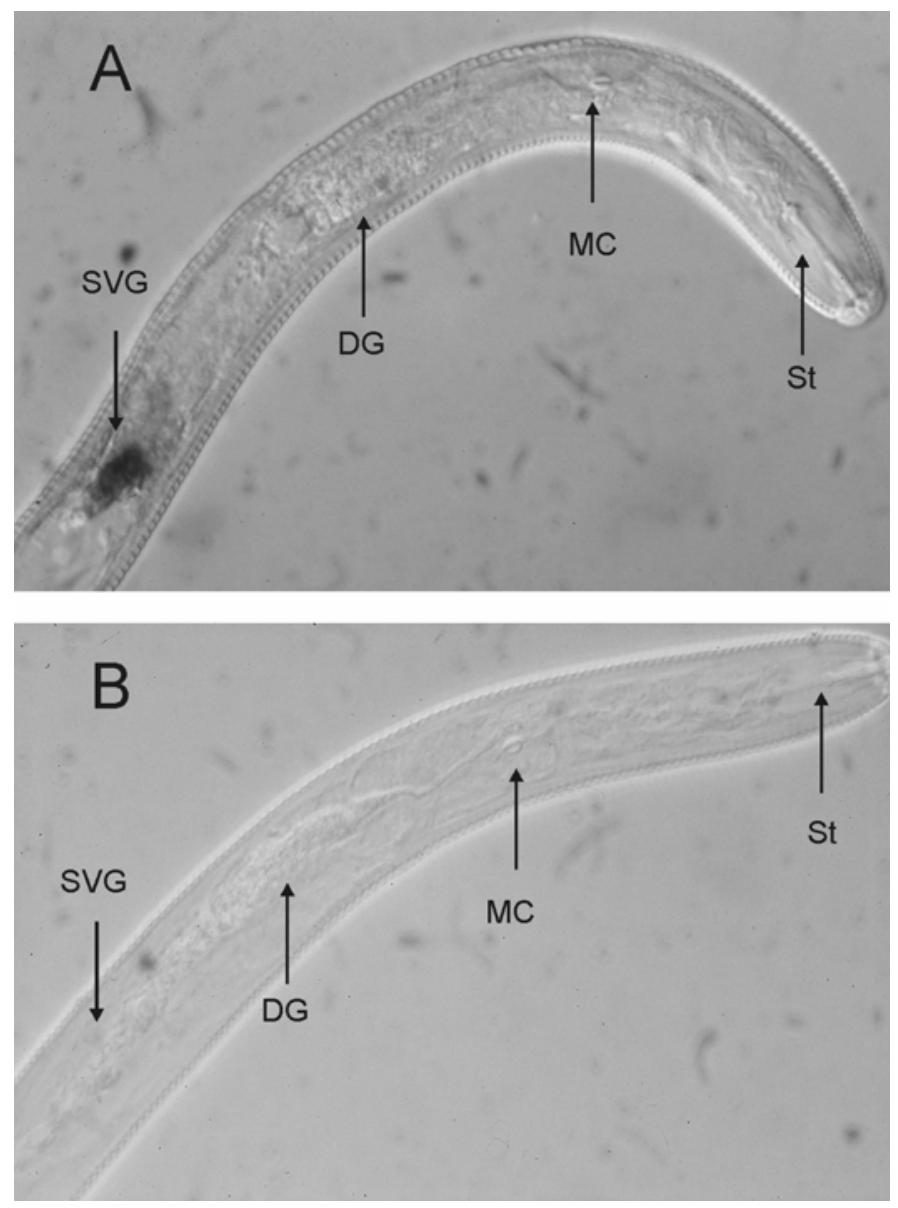

Fig. 2. In situ hybridization microscopy of Gr-eng $3 / 4$ transcripts using digoxigenin-labeled sense and antisense probes amplified from Gr-eng-3/4 cDNA in sections of second-stage preparasitic juveniles of Globodera rostochiensis. A, The antisense probe specifically hybridized in the posterior tip of the subventral esophageal gland (SVG) lobe. B, No hybridization could be observed with the corresponding sense probe. A and B, The dorsal gland (DG), metacorpus (MC), and stylet (St) are indicated with arrows.
Two positions with introns in Gr-engl and Gr-eng2 (V and VII) are not used in Gr-eng3 and Gr-eng4. In total, the regions coding for the GHF5 domain in Gr-eng1 and Gr-eng2 include seven introns. The introns at position I differ in size by 83 nucleotides between the Gr-eng3 and Gr-eng4 genes (354 and 271 nucleotides for Gr-eng-3 and Gr-eng-4, respectively). The other introns (at positions II, III, IV, VI, and VIII) in Gr-eng3 and Gr-eng4 differ only at a small number of nucleotide positions and are of similar sizes. The nucleotide substitutions at positions 64,65 , 172, 275, and 949 of the coding sequence cause substitution of four amino acids $\left(\mathrm{K}_{21} \rightarrow \mathrm{L}_{21}, \mathrm{D}_{56} \rightarrow \mathrm{Y}_{56}, \mathrm{~N}_{91} \rightarrow \mathrm{K}_{91}\right.$, and $\left.\mathrm{T}_{316} \rightarrow \mathrm{A}_{316}\right)$. The three other polymorphisms do not lead to differences in the amino acid composition of the encoded proteins.

An antisense digoxigenin-11-dUTP-labeled cDNA probe was constructed for in situ hybridization microscopy to localize the expression of Gr-eng 3 and Gr-eng4 in nematodes. The high similarity between Gr-eng3 and Gr-eng4 did not allow us to design probes specific for each individual gene. The antisense probe specifically hybridized in both subventral esophageal gland cells in preparasitic juveniles of G. rostochiensis (Fig. 2A). The sense probe did not hybridize to structures in the nematode (Fig. 2B). Therefore, expression of Gr-eng3 and Gr-eng4 is restricted to the subventral esophageal gland cells.

Further analysis of the protein sequence using the PFAM HMM search program showed that Gr-ENG3 and Gr-ENG4 comprise a single domain protein (amino acids 37-297) which is recognized as a glycosyl hydrolase family 5 cellulase (GHF 5; $P$ value of $10^{-38}$ ). In this domain, two conserved regions, IYEIWNE ${ }_{163} \mathrm{P}$ and FVTE $_{253}$ YGI, including two active site glutamic acid residues were identified in Gr-ENG3 and Gr-ENG4 (14). To test whether the Gr-ENG3 and Gr-ENG4 proteins have cellulase activity, we subcloned the predicted open reading frames into pET101 for recombinant expression in bacterial cells. Cell lysates of transformed E. coli containing Gr-eng3 and Gr-eng4 constructs showed significant hydrolytic activity in a cup plate assay with $0.2 \%$ carboxymethylcellulose (Fig. 3B and C). The lysates of $E$. coli transformants that were not induced to express the protein (data not shown) or that harbored the empty plasmid vector did not show such activity (Fig. 3A). Based on these results, we conclude that Gr-ENG3 and Gr-ENG4 encode functional cellulases.

The monoclonal antibody MGR48 recognizes at least four proteins encoding cellulase activity of which Gr-ENG3 and GrENG4 were shown to be the most abundant in stylet secretions (26). Chen et al. (6) found that using RNAi to knock-down expression of Gr-eng1 and Gr-eng2 reduced infectivity of the nematodes by approximately $50 \%$. To investigate the relative importance of Gr-eng3/4 for nematode infectivity, we knockeddown these genes in preparasitic juveniles by soaking in specific dsRNA (Fig. 4). Again, the high similarity between Gr-eng3 and Gr-eng4 did not allow us to target each gene separately. The nematodes were soaked in dsRNA specifically designed on $G r$ eng3/4, and off-target effects were not detectable with RT-PCR (Fig. 4). After soaking, the juveniles were transferred to in vitrocultured potato roots (Solanum tuberosum Line V), and penetration and intracellular migration was assessed at $24 \mathrm{~h}$ post-
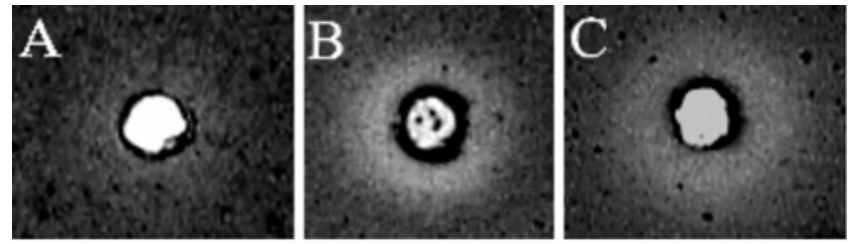

Fig. 3. A cup plate enzyme activity assay showing the hydrolytic activity on carboxymethylcellulose of $\mathbf{B}$, Gr-ENG3 and $\mathbf{C}$, Gr-ENG4 recombinantly expressed in Escherichia coli. The hydrolytic activity in the substrate is visualized as a halo by a Congo Red stain. A, No halo could be detected while applying lysates of $E$. coli harboring the empty plasmid. 
inoculation (experiment was repeated three times with different batches of nematodes). The proportion of nematodes inside the roots, calculated relative to the number of nematodes transferred to the roots, was $10.38 \%$ ( $\mathrm{SD} \pm 6.01$ ) for nematodes treated with Gr-eng3/4 dsRNA, whereas $23.57 \%$ (SD \pm 5.79 ) of the nematodes from the control treatment penetrated the roots at $24 \mathrm{~h}$ postinoculation (Table 2). We further studied the effect of such a significant reduction of infectivity $(56.6 \% ; P$ value in Student's $t$ test of 0.0195 ) on penetration behavior of the nematodes. Following silencing of Gr-eng3/4, we observed a high proportion $(\approx 77 \%)$ of the penetrating nematodes partially inside the root, i.e., with only the anterior segment of the nematode including the esophageal gland region in the epidermis and subepidermal layers (Fig. 5A and B). In control nematodes, partial penetration of the roots by nematodes was rare $(\approx 2 \%$; Fig. $5 \mathrm{~A}$ and $\mathrm{D})$. We also observed many small brown spots consisting of a number of epidermis cells on roots inoculated with Gr-eng3/4 dsRNA-treated nematodes. These spots seem to arise from cells in the root epidermis damaged by nematodes attempting to penetrate the root at a specific site. These nematodes apparently failed to enter the root and subsequently moved on to another site on the root (Fig. $5 \mathrm{~B}$ and $\mathrm{C}$ ). The spots are rarely observed in roots inoculated with nematodes exposed to the control treatments. Root penetration of dsRNA-treated nematodes was further assessed at 7 days postinoculation, resulting in similar outcomes (data not shown). None of these specific observations were seen following the knockdown of nematode genes with functions not directly relating to plant cell wall modification ( $G r-G A P D H$ and $G r-A 41$; data not shown).

Recent work on the evolution of nematode GHF5 cellulases based on the comparison of the coding sequences and the intron/ exon structures of the genes suggests that the variants of nematode cellulases found to date arose through sequential loss of the ancillary C-terminal domains (17). To test this "sequential loss" hypothesis we used all presently known cellulases from cyst nematodes to infer the evolutionary history of the GHF5 domain with a Bayesian analysis (Fig. 6). In the resulting tree, the length of the horizontal branches reflects the accumulated changes in the GHF5 domain in cyst nematode cellulases since the last common ancestor genes (the nodes). Our analysis indicated that there are at least two distinct evolutionary lineages of cellulases in cyst nematodes (clades I and II) originating from two distinct ancestor GHF5 genes (CelI and CelII). The sequence of descent of CelI and CelII from the "Ur"-cellulase $\left(\mathrm{Cel}^{*}\right)$ cannot be reliably

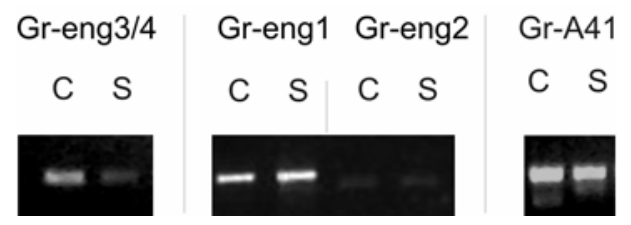

Fig. 4. Semiquantitative reverse transcription-polymerase chain reaction showing the expression of A, Gr-eng3/4, B, Gr-eng1 (1,116 bp), C, Gr-eng2 (1,029 bp), and D, Gr-A41 (660 bp) in preparasitic second-stage juveniles of Globodera rostochiensis following a control treatment (C) and treatment with Gr-eng3/4-specific dsRNA (S). The samples shown here are taken from the reaction mix after 26 cycles of polymerase chain reaction. resolved with the information captured in currently available sequences. For each cellulase, we indicated in the tree the protein domain structure out of three possible variants found in nematodes so far: (i) a GHF5 domain with a glycine/serine-rich linker sequence and a type II cellulose binding domain (GHF5+LSG+CBDII as in Gr-ENG1); (ii) a GHF5 domain with a so-called highly charged AKP motif (GHF5+AKP as in Gr-ENG2) similar to linker histones H1 and H5 (GenBank accession AF107026 and PFAM accession PF00538 [15]); and (iii) a GHF5 domain alone (GHF5 as in Gr-ENG3/4). The sequential loss-hypothesis is supported when GHF5+L-SG+CBDII, GHF5+L-SG, and/or GHF5 variants appear in a single monophyletic clade with, ideally, the GHF5+L-SG+CBDII variant being most basal and the GHF5 variant as the most recent speciation. Cellulases in clade II, including Gr-ENG3, Gr-ENG4, Hg-ENG5, and Hg-ENG6, do not have ancillary domains and therefore cannot be used to test the hypothesis. Clade I comprises a polytomy of three groups of cyst nematode cellulases (subclades $a, b$, and $c$ ) for which it is not possible to determine the sequence of descent from CelI, but within each of the subclades $a, b$, and $c$ the inference of the sequence of descent is robust (posterior probabilities of 0.95 or higher) and they can be used to test the hypothesis. The inferred phylogeny in these branches indicates that none of these three subclades includes all three extant domain structure variants, i.e., the GHF5+L-SG+CBDII and its two possible C-terminal truncations. The GHF5+AKP variant clusters together with the GHF5+L-SG+CBDII variant within subclade $c$, while it also is in monophyly with the GHF5 variant in subclade $b$. The GHF5+L$\mathrm{SG}+\mathrm{CBDII}$ variant is not found in the same subclade as the GHF5-variant, while it is in monophyly with the GHF5+AKP variant in subclade $c$. Furthermore, the sequential loss hypothesis assumes that the L-SG linker and the AKP-domain are homologous (Fig. 7). However, while using the BLAST2SEQ option at the NCBI to make a pair wise comparison between all possible combinations of the L-SG and the AKP sequences, none showed significant similarity (E values $>10$ ). Furthermore, it should be noted that the GHF5+L-SG variant has not been found in nematodes to date and that the analysis of these gene families is still limited by the total amount of available sequence information. However, in the currently available data set, support for the contribution of sequential loss in the evolution of domain variants in cyst nematodes is limited. Genome projects for a range of plant-parasitic nematodes including $M$. incognita, M. hapla, and G. pallida will help resolve the detailed evolution of the cellulase gene family.

\section{DISCUSSION}

At the onset of parasitism, infective nematodes penetrate the host plant to migrate toward the vascular cylinder to establish a permanent feeding site. Plant penetration results from the mechanical impact of stylet thrusts and chemical weakening of the cell wall by nematode proteins secreted through the stylet. The monoclonal antibody MGR48 recognizes at least three proteins in stylet secretions of the potato cyst nematode $G$. rostochiensis of which two were found to be cellulases in a previous study. In this paper, we report on the identification and functional analysis

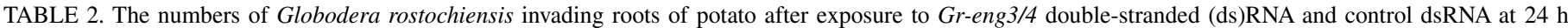
postinoculation $^{\mathrm{a}}$

\begin{tabular}{|c|c|c|c|c|c|}
\hline Treatment & $\begin{array}{l}\text { Number of nematodes } \\
\text { used for inoculation }\end{array}$ & $\begin{array}{l}\text { Number partially } \\
\text { inside root }\end{array}$ & $\begin{array}{l}\text { Number completely } \\
\text { penetrated root }\end{array}$ & $\begin{array}{c}\text { Total number of } \\
\text { penetrating nematodes }\end{array}$ & $\begin{array}{c}\text { Percentage } \\
\text { penetrating nematodes }\end{array}$ \\
\hline Control dsRNA & $175 \pm 33$ & 1 & 40 & $41 \pm 13$ & $23.57 \pm 5.7$ \\
\hline Gr-eng3/4 dsRNA & $175 \pm 29$ & 14 & 4 & $18 \pm 10$ & $10.38 \pm 6.0$ \\
\hline
\end{tabular}

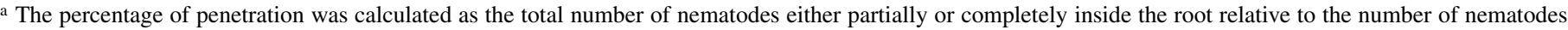
inoculated. The infection assay was repeated three times with four replicates per treatment and four root systems per replicate. The probability that the difference between both treatments is not significant is 0.0097 ( $P$ value, in a one-tail $t$ test while assuming equal variances; \pm indicates standard deviation). 
of two novel cellulase genes encoding the most abundant antigens recognized by MGR48 in the stylet secretions of G. rostochiensis. Remarkably, the overall identity between the four proteins that evidently have the epitope of MGR48 in common is only $42 \%$.

The four cellulase genes from G. rostochiensis (Gr-engl, Greng2, Gr-eng3, and Gr-eng4) are specifically expressed in the subventral esophageal glands of the nematodes. Several studies dating back to the 1980 s reported on the presence of intracellular Rickettsia-like bacteria in these esophageal gland cells (30), which suggests that they could contribute to the cellulolytic system of the nematodes in a manner analogous to the situation in termites. However, the cellulase genes described here, and all others described from nematodes to date, show features typical of eukaryotic genes (e.g., poly-A tails and introns). At present, there is no evidence that intracellular eukaryotic microbes inhabit the esophageal glands of cyst nematodes and we therefore conclude that these cellulases are of nematode origin.

Cyst nematodes are far more damaging to host tissues during migration than root-knot nematodes, suggesting that the cellulolytic system in cyst nematodes may be less advanced rendering the nematode more dependent on physical impact of stylet thrusts (12). However, as the cellulolytic system in cyst nematodes unfolds further with each new finding, it appears to be elaborate involving proteins with variety of domain configurations. The configurations known to date comprise a glycosyl hydrolase catalytic domain, a linker rich in serine and glycine, an AKP motif, a bacterial type II cellulose binding domain, and an expansin domain. The glycosyl hydrolase domains exhibit cellulose activity on a variety of substrates, including cellulose and hemicellulose polymers (9). The L-SG linkers appear between GHF5 and CBDII, whereas the AKP motif is present at the $\mathrm{C}$ terminus of GHF5 domains. The AKP motif, whose role remains to be determined experimentally, consists of between 25 and 43 amino acids and is found in Gr-ENG2, Gts-ENG2, Hs-ENG2, and Hg-ENG4, and shows strong similarity with linker histones H1 and H5 (e.g., GenBank accession AF107026). The highly charged AKP helix in linker histones is involved in protein-protein and protein-DNA interactions within aggregated nucleosomes (15). It is possible that the AKP motif in cyst nematode cellulase is an evolutionary remnant that once linked the GHF5 domain to a type II cellulose binding domain. However, this $\mathrm{C}$-terminal peptide sequence may also serve to aggregate other nematode enzymes and proteins into a cellulolytic complex. Anaerobic microbes have evolved such high-molecular weight complexes of multiple cellulases and hemicellulases (2). The enzymes aggregated into these so-called cellulosomes have synergetic activity on insoluble cellulose in plant cell walls. Nematodes also secrete a range of cellulose degrading enzymes that are believed to collectively breakdown plant cellulose. The AKP domain in cyst nematode cellulases may facilitate the assembly of the GHF5 domain into a complex with other cellulose modifying proteins (e.g., hydrolytic enzymes and expansins).

Bayesian inference of the phylogeny of cyst nematode cellulases combined with the projection of domain structure onto the tree revealed a lack of correlation between the evolutionary history of the GHF5 domain and a sequential loss of the Cterminal CBDII domain and the L-SG linker. Support for the sequential loss-hypothesis would have been provided if the GHF5+L-SG+CBDII variants and the two possible truncates clustered together in a monophyletic clade. However, the theoretical intermediate GHF5+L-SG has not been found in plantparasitic nematodes to date. The extant cellulase variant that resembles GHF5+L-SG the most includes a GHF5 and a C-terminal AKP motif (e.g., Gr-ENG2). The linker L-SG and the highly charged C-terminal AKP motif are not similar in sequence, indicating that both domains are not homologous traits. Therefore, the GHF5+AKP variant is not a likely evolutionary intermediate between the GHF5+L-SG+CBD and the GHF5 variants. We also did not find support for the proposal that the extant GHF5 variants in cyst nematodes arose, as an evolutionary innovation, from an extinct ancestor gene coding for GHF5+L-SG. Nor did we find sufficient support for an ancestor gene that derives from an ancient cellulase that combined a GHF5 domain, a linker, and a CBDII (domain). In contrast, the currently available data suggest that the extant nematode cellulases arose through reshuffling of GHF5 domain with different types of ancillary domains as

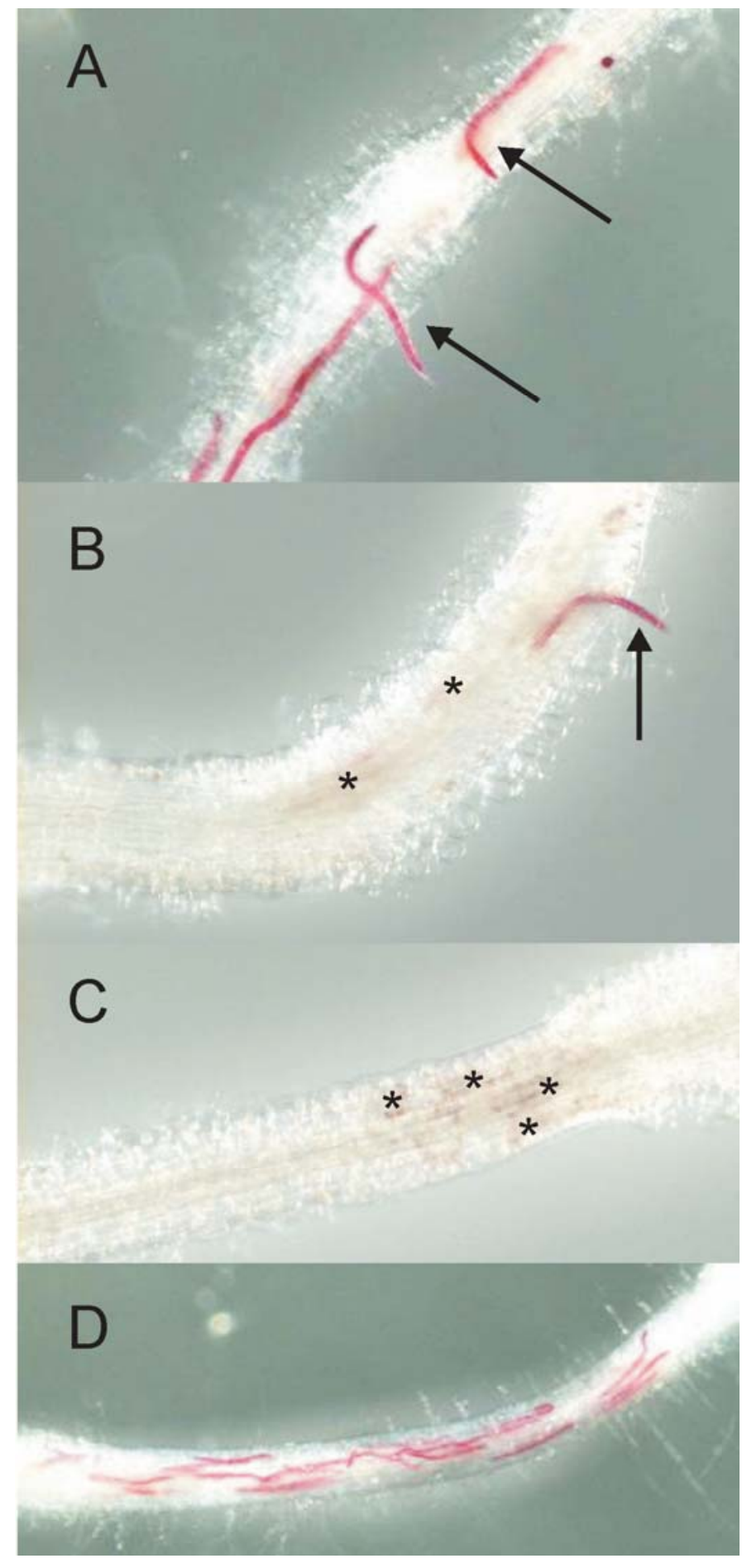

Fig. 5. A, B, and C, Infections of in vitro cultured potato roots with Globodera rostochiensis second-stage juveniles following Gr-eng3/4 dsRNA treatment and $\mathbf{D}$, control treatment $24 \mathrm{~h}$ postinoculation. The roots are stained with acid fuchsin to visualize the nematodes in red. Nematodes that achieved partial penetration are indicated with arrows. Brownish spots of damaged epidermis cells are indicated with an asterisk (*). 


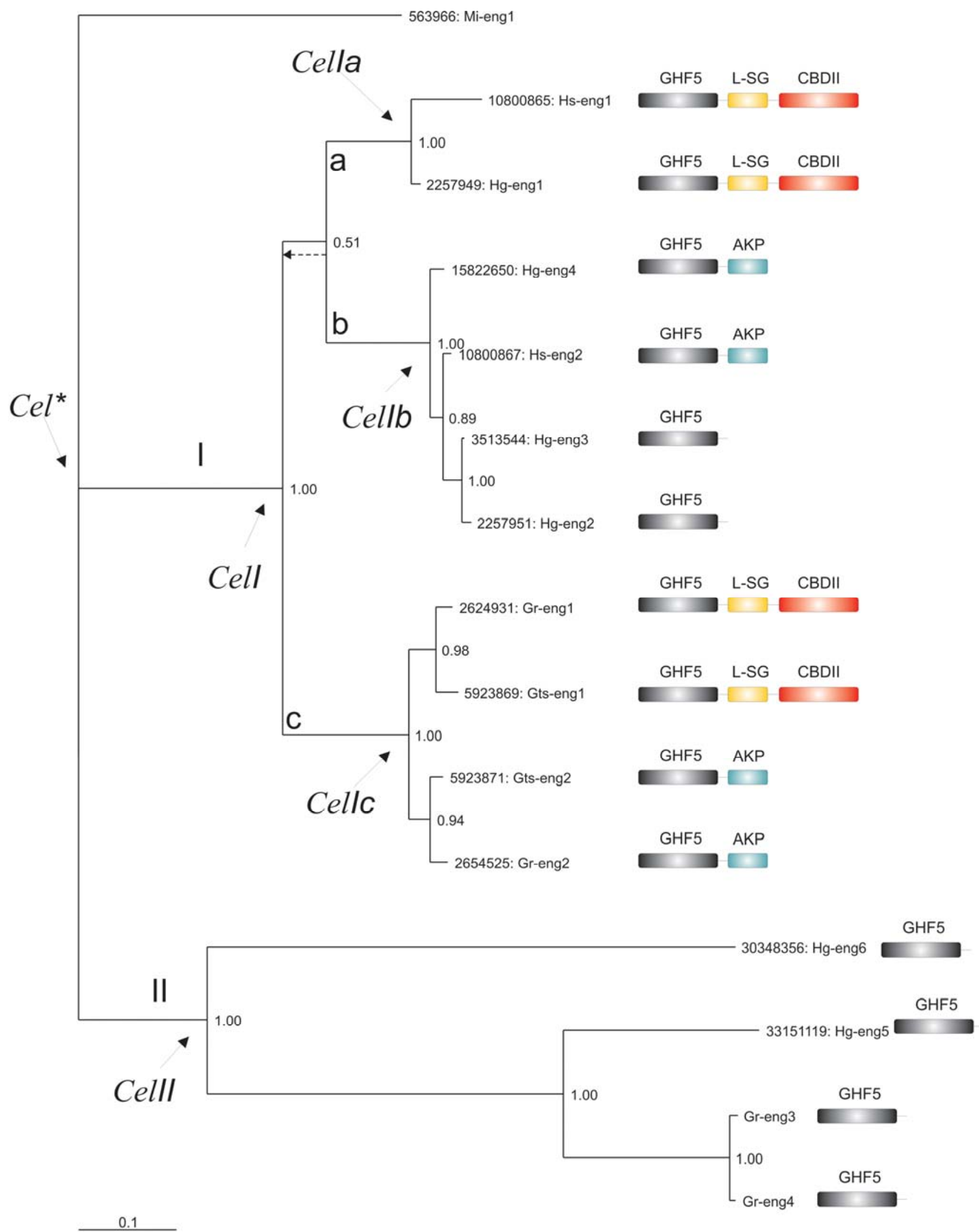

Fig. 6. A rooted tree based on a Bayesian inference of phylogeny of 14 GHF5 cellulases from four cyst nematodes species with significant similarity to Gr-ENG3 and Gr-ENG4. The numbers at the nodes indicate the posterior probabilities as calculated in MrBayes. The root-knot nematode GHF5 cellulase Mi-ENG1 was included in the analysis to root the resulting tree. The protein domain structure out of three possible variants is included for each of the taxa (GHF5, glycosyl hydrolase family 5; L-SG, linker region rich in serine and glycine residues; AKP, short highly charged stretch with similarity to linker histone 1- and 5-like AKPmotifs; CBDII, bacterial type 2 cellulose binding domain). The nematode cellulases are indicated with their protein names (Hs, Heterodera schachtii; Hg, Heterodera glycines; Gr, Globodera rostochiensis; Gts, Globodera tabacum subsp. solanacearum; and Mi, Meloidogyne incognita), and their GenBank identification numbers. Robust monophyletic clades (I and II) and inferred ancestor genes coding for GHF5 cellulase ( $\mathrm{Cel}$ ) are indicated along the branches and the nodes. The arrows points at the unresolved polytomy of three subclades $(\mathrm{a}, \mathrm{b}$, and $\mathrm{c}$ ) within lineage $\mathrm{I}$. 


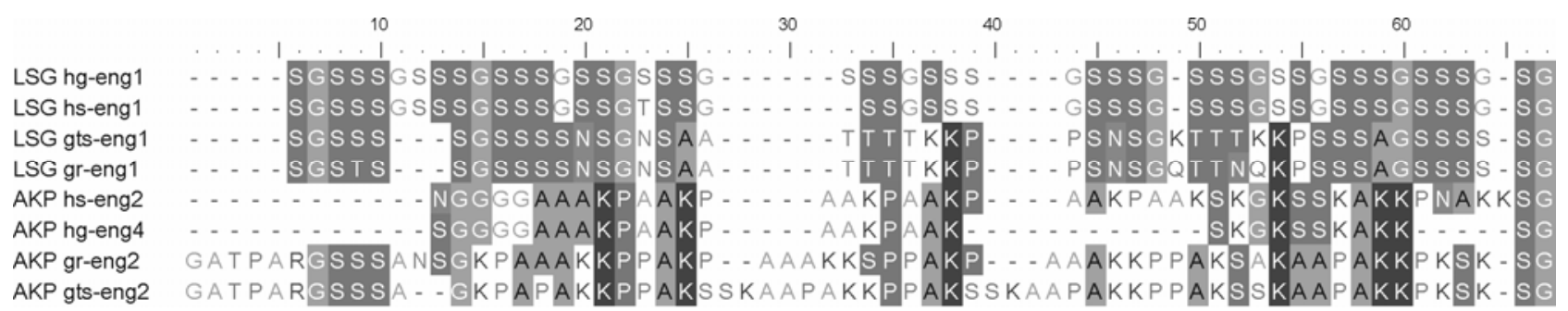

Fig. 7. A protein sequence alignment of the LSG linker sequences and the AKP domain in GHF5 cellulases from cyst nematodes made in ClustalW.

relatively independent units. The fact that highly similar cellulose binding domains occur in proteins with entirely different sequences and activities further supports this model (e.g., the CBDII in Gr-ENG1 and in the $\beta$-expansin Gr-EXP1 have 52\% identity and $69 \%$ similarity).

To study the relative importance and the role of Gr-eng3 and Gr-eng4 in parasitism of G. rostochiensis, we monitored plant penetration and intracellular migration of infective juveniles in which the genes were knocked-down by RNA interference. Rosso et al. (23) reported for $M$. incognita that RNA interference by soaking in dsRNA is transitory, and may last not longer than a few days. Recent studies, however, suggest that the effects may last considerably longer in cyst nematodes (1). For both cyst and rootknot nematodes, soaking in a highly concentrated solution of dsRNA is clearly a valuable method for studying genes involved at the initial stages of parasitism in plants, and we demonstrate this again in this study. Knocking-down expression of Gr-eng3 and Gr-eng4 in preparasitic nematodes reduced the penetration rate of plants by the nematodes by more than $50 \%$. Chen et al. (6) observed some down regulation of Gr-eng3/4 when they targeted Gr-engl with RNAi, suggesting that in their experiment reduced levels of Gr-eng3/4 could have affected nematode infectivity. We have seen no such off-target silencing effect on the expression of Gr-eng1 and Gr-eng2 in our experiments. Therefore, the reduced penetration observed here is attributed to removal of Gr-eng3 and Gr-eng4.

Knocking-down Gr-engl and Gr-eng 2 by RNA interference resulted in a significant reduction in infectivity as was measured 2 weeks after inoculation, but the mechanism underlying this phenotype was not clear (6). Our current observations of the spots of damaged epidermis cells on host plant roots and the incapacity to achieve full penetration of $G r$-eng3/4 dsRNA-treated nematodes show that poor invasion of the host plants is the probable cause of the decline in infectivity.

\section{ACKNOWLEDGMENTS}

This work was funded by the NWO 'Vernieuwingsimpuls' (G. Smant and S. Rehman) and by RERAD Potato Pathology Workpackage (WP1.5) (J. Jones).

\section{LITERATURE CITED}

1. Bakhetia, M., Urwin, P. E., and Atkinson, H. J. 2007. qPCR analysis and RNAi define pharyngeal gland cell-expressed genes of Heterodera glycines required for initial interactions with the host. Mol. Plant-Microbe Interact. 20:306-312.

2. Bayer, E. A., Belaich, J. P., Shoham, Y., and Lamed, R. 2004. The cellulosomes: Multienzyme machines for degradation of plant cell wall polysaccharides. Annu. Rev. Microbiol. 58:521-554.

3. Bird, A. F. 1983. Changes in the dimensions of the esophageal glands in root-knot nematodes during the onset of parasitism. Int. J. Parasitol. 54:879-890.

4. Carpita, N. C. 1996. Structure and biogenesis of the cell walls of grasses. Annu. Rev. Plant Physiol. Plant Mol. Biol. 47:445-476.

5. Carpita, N. C., and Gibeaut, D. M. 1993. Structural models of primary cell walls in flowering plants: Consistency of molecular structure with the physical properties of the walls during growth. Plant J. 3:1-30.

6. Chen, Q., Rehman, S., Smant, G., and Jones, J. T. 2005. Functional analysis of pathogenicity proteins of the potato cyst nematode Globodera rostochiensis using RNAi. Mol. Plant-Microbe Interact. 18:621.

7. De Boer, J. M., Overmars, H. A., Bakker, J., and Gommers, F. J. 1992. Analysis of two-dimensional protein patterns from developmental stages of the potato cyst nematode, Globodera rostochiensis. Parasitology 105:461-474.

8. De Boer, J. M., Yan, Y., Smant, G., Davis, E. L., and Baum, T. J. 1998. Insitu hybridization to messenger RNA in Heterodera glycines. J. Nematol. 30:309-312.

9. Gao, B. L., Allen, R., Davis, E. L., Baum, T. J., and Hussey, R. S. 2004. Developmental expression and biochemical properties of a beta-1,4endoglucanase family in the soybean cyst nematode, Heterodera glycines. Mol. Plant Pathol. 5:93-104.

10. Goellner, M., Smant, G., De Boer, J. M., Baum, T. J., and Davis, E. L. 2000. Isolation of beta-1,4-endoglucanase genes from Globodera tabacum and their expression during parasitism. J. Nematol. 32:154-165.

11. Goverse, A., van der Voort, J. R., van der Voort, C. R., Kavelaars, A., Smant, G., Schots, A., Bakker, J., and Helder, J. 1999. Naturally induced secretions of the potato cyst nematode co-stimulate the proliferation of both tobacco leaf protoplasts and human peripheral blood mononuclear cells. Mol. Plant-Microbe Interact. 12:872-881.

12. Hansen, E., Harper, G., McPherson, M. J., and Atkinson, H. J. 1996. Differential expression patterns of the wound-inducible transgene wun1uidA in potato roots following infection with either cyst or root knot nematodes. Physiol. Mol. Plant Pathol. 48:161-170.

13. Jaubert, S., Laffaire, J. B., Abad, P., and Rosso, M. N. 2002. A polygalacturonase of animal origin isolated from the root-knot nematode Meloidogyne incognita. FEBS Lett. 522:109-112.

14. Jenkins, J., Leggio, L. L., Harris, G., and Pickersgill, R. 1995. $\beta$ Glucosidase, $\beta$-galactosidase, family A cellulases, family $\mathrm{F}$ xylanases and two barley glycanases form a superfamily of enzymes with 8 -fold, $\beta / \beta$ architecture and with two conserved glutamates near the carboxy-terminal ends of $\beta$-strands four and seven. FEBS Lett. 362:281-285.

15. Kasinsky, H. E., Lewis, J. D., Dacks, J. B., and Ausio, J. 2001. Origin of H1 linker histones. FASEB J. 15:34-42.

16. Kudla, U., Qin, L., Milac, A., Kielak, A., Maissen, C., Overmars, H., Popeijus, H., Roze, E., Petrescu, A., Smant, G., Bakker, J., and Helder, J. 2005. Origin, distribution and 3D-modeling of Gr-EXPB1, an expansin from the potato cyst nematode Globodera rostochiensis. FEBS Lett. 57:2451-2457.

17. Ledger, T. N., Jaubert, S., Bosselut, N., Abad, P., and Rosso, M. N. 2006. Characterization of a new beta-1,4-endoglucanase gene from the rootknot nematode Meloidogyne incognita and evolutionary scheme for phytonematode family 5 glycosyl hydrolases. Gene 382:121-128.

18. Mateos, P. F., Jimenez Zurdo, J. I., Chen, J., Squartini, A. S., Haack, S. K., Martinez Molina, E., Hubbell, D. H., and Dazzo, F. B. 1992. Cellassociated pectinolytic and cellulolytic enzymes in Rhizobium leguminosarum biovar trifolii. Appl. Environ. Microbiol. 58:1816-1822.

19. Mitreva-Dautova, M., Roze, E., Overmars, H., De Graaff, L., Schots, A., Helder, J., Goverse, A., Bakker, J., and Smant, G. 2006. A symbiontindependent endo-1,4- $\beta$-xylanase from the plant-parasitic nematode Meloidogyne incognita. Mol. Plant-Microbe Interact. 19:521-529.

20. Popeijus, H., Overmars, H., Jones, J., Blok, V., Goverse, A., Helder, J., Schots, A., Bakker, J., and Smant, G. 2000. Enzymology-Degradation of plant cell walls by a nematode. Nature 406:36-37.

21. Qin, L., Kudla, U., Roze, E. H. A., Goverse, A., Popeijus, H., Nieuwland, J., Overmars, H., Jones, J. T., Schots, A., Smant, G., Bakker, J., and Helder, J. 2004. Plant degradation: A nematode expansin acting on plants. Nature 427:30.

22. Ronquist, F., and Huelsenbeck, J. P. 2003. MrBayes 3: Bayesian phylogenetic inference under mixed models. Bioinformatics 19:15721574.

23. Rosso, M. N., Dubrana, M. P., Cimbolini, N., Jaubert, S., and Abad, P. 2005. Application of RNA interference to root-knot nematode genes encoding esophageal gland proteins. Mol. Plant-Microbe Interact. 18:615.

24. Rosso, M. N., Favery, B., Piotte, C., Arthaud, L., De Boer, J. M., Hussey, 
R. S., Bakker, J., Baum, T. J., and Abad, P. 1999. Isolation of a cDNA encoding a beta-1,4-endoglucanase in the root-knot nematode Meloidogyne incognita and expression analysis during plant parasitism. Mol. Plant-Microbe Interact. 12:585-591.

25. Sambrook, J., Fritsch, E. F., and Maniatis, T. 1989. Molecular Cloning: A Laboratory Manual. Cold Spring Harbor Laboratory, Cold Spring Harbor, NY.

26. Smant, G., Goverse, A., Stokkermans, J. P. W. G., De Boer, J. M., Pomp, H., Zilverentant, J. F., Overmars, H. A., Helder, J., Schots, A., and Bakker, J. 1997. Potato root diffusate induced secretion of soluble, basic proteins originating from the subventral esophageal glands of potato cyst nematodes. Phytopathology 87:839-845.

27. Smant, G., Stokkermans, J. P. W. G., Yan, Y., De Boer, J. M., Baum, T. J., Wang, X., Hussey, R. S., Gommers, F. J., Henrissat, B., Davis, E. L., Helder, J., Schots, A., and Bakker, J. 1998. Endogenous cellulases in animals: Isolation of $\beta-1,4$-endoglucanase genes from two species of plant-parasitic cyst nematodes. Proc. Natl. Acad. Sci. USA 95:4906-4911.
28. Sulston, J., and Hodgkin, J. 1988. Methods. In The Nematode Caenorhabditis elegans. W. B. Wood, ed. Cold Spring Harbor Laboratory, Cold Spring Harbor, NY.

29. Uehara, T., Kushida, A., and Momota, Y. 2001. PCR-based cloning of two beta-1,4-endoglucanases from the root-lesion nematode Pratylenchus penetrans. Nematology 3:335-341.

30. Walsh, J. A., Shepherd, A. M., and Lee, D. L. 1983. The distribution and effect of intracellular rickettsia-like micro-organisms infecting secondstage juveniles of the potato cyst-nematode Globodera rostochiensis. J. Zool. 199:395-419.

31. Wang, X., Meyers, D., Yan, Y., Baum, T. J., Smant, G., Hussey, R. S., and Davis, E. L. 1999. In planta localization of a beta-1,4-endoglucanase secreted by Heterodera glycines. Mol. Plant-Microbe Interact. 12:64-67.

32. Yan, Y. T., Smant, G., and Davis, E. 2001. Functional screening yields a new beta-1,4-endoglucanase gene from Heterodera glycines that may be the product of recent gene duplication. Mol. Plant-Microbe Interact. 14:63-71. 